\title{
CHARACTER THEORY AND ROOTED MAPS IN AN ORIENTABLE SURFACE OF GIVEN GENUS: FACE-COLORED MAPS
}

\author{
D. M. JACKSON AND T. I. VISENTIN
}

\begin{abstract}
The character theoretic approach [5] to the enumeration of rooted maps in an orientable surface of arbitrary genus is extended to 2-face-colorable rooted maps. In particular, we show that there exists, for each genus, a correspondence between the set of 2-colored triangulations and a set of 2-colored rooted maps of all lower genera with a distinguished subset of vertices.
\end{abstract}

\section{INTRODUCTION}

A previous paper [5] was concerned exclusively with the combinatorial interpretation of products of pairs of permutations, one of which is a fixed point free involution, in which information has been retained about their number of cycles. It was seen that an appropriate context is the embedding of rooted graphs in orientable surfaces. It is therefore natural to seek an analogous combinatorial interpretation of products of pairs of permutations with no such restriction and, more generally still, products of a finite number of them, with a similar retention of information about the number of cycles in each.

In $\S 2$ we define a permutation system, an extension of the rotation systems used in [5] to a product of a finite number of prescribed conjugacy classes, and give their connection with embeddings of 2-face-colorable maps. Throughout this paper, all surfaces are oriented and without boundaries. Generating functions for certain subclasses of these maps are derived in $\S 3$. Factorizations for permutation systems are given in $\S 4$ and are used in $\S 5$ to obtain two bijections. These are between rooted 2-colored maps with all root-colored faces having degree $r$ and $2 r$-face-regular maps and between 2-colored rooted triangulations and 2-colored rooted maps.

Necessarily, extensive use is made of character theoretic results stated in [5]. However, references to this paper have been confined, in the main, to the proofs of a few character theoretic results on which this extension rests. Notation and terminology are those used in [5], to which the reader is therefore referred for

Received by the editors December 1, 1988.

1980 Mathematics Subject Classification (1985 Revision). Primary 05A15, 20C15; Secondary $57 \mathrm{~N} 37$.

This work was supported by a grant from the Natural Sciences and Engineering Research Council of Canada (A-8235). 
basic definitions, such as those for embeddings and rotation systems, which are not duplicated here. For further details concerning combinatorial topology, the algebra of symmetric functions and representation theory, the reader may consult [3], [6], and [7], respectively. The notation which is used throughout the paper is given below.

For an integer $n>0$, we write $\theta \vdash n$ to indicate that $\theta$ is a partition of $n$. The partition $(p, \ldots, p) \vdash k p$ is denoted by $\left[p^{k}\right]$. The conjugate of $\theta$ is denoted by $\tilde{\theta}$. For $\mathscr{A} \subseteq \mathscr{N}=\{1,2, \ldots\}, \Pi_{\mathscr{A}}$ denotes the set of all partitions with no part not in $\mathscr{A}$. The set $\{\theta \vdash n\}$ is a natural index set for the conjugacy classes of $\mathfrak{S}_{n}$, the symmetric group on $n$ symbols. The conjugacy class corresponding to $\theta$ is denoted by $\mathscr{C}_{\theta}$. The order of $\mathscr{C}_{\theta}$ is $h^{\theta}=n ! g(\theta)$ where $g(\theta)=\prod_{i \geq 1}\left(i^{m_{i}} m_{i} !\right)^{-1}$.

The group algebra of $\mathfrak{S}_{n}$ over the complex field $\mathbf{C}$ is denoted by $\mathrm{CS}_{n}$. Multiplication of permutations is carried out from right to left. A permutation in $\mathfrak{S}_{k p}$ is $p$-semiregular if its cycle-type is $\left[p^{k}\right]$. Occasionally, we drop the prefix and refer to such permutations as semiregular.

$\chi^{\theta}$ denotes the irreducible (ordinary) character associated with $\mathscr{C}_{\theta}$ and $\chi_{\alpha}^{\theta}$ denotes its value at any element of $\mathscr{C}_{\alpha}$ where $\alpha, \theta \vdash N$. We adopt the convention that the character associated with the empty partition is identically 1 and that $\chi_{\alpha}^{\theta}=0$ if $|\theta| \neq|\alpha|$. The degree, $f^{\theta}$, of the irreducible representation indexed by $\theta$ is equal to $\chi_{\left[1^{N}\right]}^{\theta}$.

The rising and falling factorial functions are, respectively,

$$
(x)^{(n)}=x(x+1) \cdots(x+n-1)
$$

and

$$
(x)_{n}=x(x-1) \cdots(x-n+1) .
$$

Finally, $H_{\theta}(x)$ denotes the polynomial $\prod_{1 \leq i \leq l(\theta)}(x-i+1)^{\left(\theta_{i}\right)}$.

Throughout, $u, x, y, z$ are commutative indeterminates. $M_{\mathscr{A}}(u, x, y, z)$ is the generating function for rooted maps with respect to genus and the numbers of vertices, faces, and edges marked, respectively, by $u, x, y, z$ and having no faces of degree not in $\mathscr{A}$. Let $\epsilon_{n}$ denote a prescribed fixed point free involution in $\mathfrak{S}_{2 n}$. Then $R_{\mathscr{A}}(x, y \mid z)$ is the generating function for the rotation systems $\nu \in \mathfrak{S}_{2 n}$ with respect to the numbers of cycles in $\nu, \nu \epsilon_{n}$, and $n$ marked, respectively, by $x, y, z$, such that $\nu \epsilon_{n}$ has no cycles of length not in $\mathscr{A}$.

\section{Permutation systems AND the gRoup Algebra}

Let $p$ be a positive integer. Then $\sigma=\left(g_{1}, \ldots, g_{p}\right) \in \mathfrak{S}_{N}^{p}$ is a permutation system for $\pi \in \mathfrak{S}_{N}$ of type $\left(t_{1}, \ldots, t_{p}\right)$ and order $p$ if $\pi=g_{1} \cdots g_{p}$ and $t_{i}$ is the number of cycles in $g_{i}$ for $i=1, \ldots, p$. We shall refer to $t_{1}+\cdots+t_{p}$ as the number of cycles in $\sigma$. For $\psi \vdash N$, let $c_{\psi}\left(t_{1}, \ldots, t_{p}\right)$ be the number of permutation systems of type $\left(t_{1}, \ldots, t_{p}\right)$ for an arbitrary but fixed $\pi \in \mathscr{C}_{\psi}$. The generating function $A_{\psi}^{(p)}\left(x_{1}, \ldots, x_{p}\right)$ for $c_{\psi}\left(t_{1}, \ldots, t_{p}\right)$ is given [4] as follows. 
Theorem 2.1.

$$
A_{\psi}^{(p)}\left(x_{1}, \ldots, x_{p}\right)=\frac{1}{N !} \sum_{\theta \vdash N} f^{\theta} \chi_{\psi}^{\theta} H_{\theta}\left(x_{1}\right) \cdots H_{\theta}\left(x_{p}\right) .
$$

We note in passing that $A_{\left[1^{n}\right]}^{(1)}(x)=x^{n}$ and, more generally, that $A_{\psi}^{(1)}=$ $x^{l(\psi)}$, where $\psi \vdash N$, and that $A_{\left[1^{n}\right]}^{(2)}(x, y)=(x y)^{(n)}$. These are immediately verifiable through the combinatorial interpretation of permutation systems, and with the aid of Theorem 2.1 they may be rewritten immediately as character sum identities. Theorem 2.1 can also be used [4] in conjunction with the material of [5] (§3) to give, for example, the number of ways of expressing a permutation as a product of a specified number of full cycles and transpositions (not necessarily adjacent ones).

A permutation system of order 2 is a generalization of a rotation system, so [5, Theorem 3.2 and Corollary 3.5] the generating function for rooted maps may be expressed as follows in terms of $A_{\left[2^{n}\right]}^{(2)}$ and the operator $\Omega_{u}$ on $\mathbf{Q}[[x, y, z]]$ defined [5] by

$$
\Omega_{u} f(x, y, z)=2 u^{2} z \frac{\partial}{\partial z} f\left(x u^{-1}, y u^{-1}, \frac{1}{2} z u\right)
$$

Theorem 2.2.

$$
\begin{gathered}
M_{\mathscr{N}}\left(u^{2}, x, y, z\right)=\Omega_{u} R_{\mathscr{N}}(x, y \mid z), \\
R_{\mathscr{N}}(x, y \mid z)=\sum_{n \geq 0} \frac{z^{n}}{n !} A_{\left[2^{n}\right]}^{(2)}(x, y) .
\end{gathered}
$$

A permutation system $(\rho, \sigma)$ on a finite set $\mathscr{U}$, such that the group generated by $\sigma$ and $\rho$ is transitive on $\mathscr{U}$, is called a hypermap [2]. A hypermap is therefore a generalization of a rotation system since the condition that one of the permutations be a fixed point free involution no longer applies. Walsh [10] exhibited a bijection between hypermaps and edge-labeled bipartite maps. This we give in its dual form after the following definitions.

A $p$-face-colorable map is a map whose faces may be assigned colors from a designated set of size $p$, called the color set, such that no edge separates faces of the same color. A $p$-face colored map is a $p$-face-colorable map with a permissible assignment of faces to colors. For example, the four color problem states that every map on the sphere is 4-face-colorable. For a rooted facecolored map, the color assigned to the root face is called the root color. For a 2-face-colored map, we may refer to the other color as the nonroot color. For a 2-face-colored map, interchange of the root-face-color corresponds to the reversal of the direction of the root edge.

Let $\mathscr{G}$ be a rooted 2 -face-colorable map, with edge set $E(\mathscr{G})=\{1, \ldots, n\}$, embedded in an orientable surface $\Sigma$. Directions are assigned to the edges of $\mathscr{G}$ so that the edges of the boundary of each face are directed consistently with the root edge. For each vertex $v$ of $\mathscr{G}$, we specify a cyclic list of origins of edges 


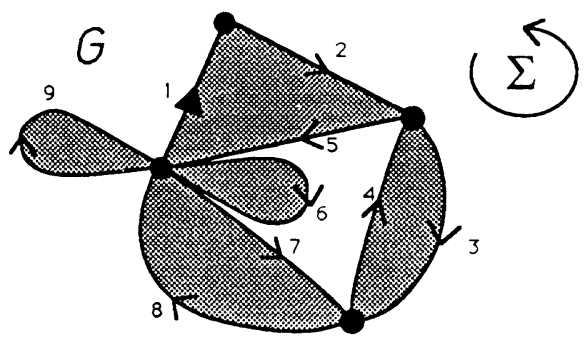

FIGURE 1. A 2-colored rooted edge-labeled map

encountered in traversing the boundary of a small disc, centered at $v$, in a sense specified by the orientation of $\Sigma$. This partitions $\{1, \ldots, n\}$ and represents a permutation $\nu \in \mathfrak{S}_{n}$. For each root-colored face $f$ of $\mathscr{G}$ we specify the edge labels encountered in traversing its boundary in a sense consistent with the orientation of $\Sigma$. This partitions $\{1, \ldots, n\}$ and represents a permutation $\phi \in \mathfrak{S}_{n}$. The corresponding permutation for nonroot-colored faces of $\mathscr{G}$ is denoted by $\epsilon$. The following bijection is the dual of Walsh's [10].

Proposition 2.3. Let $\mathscr{G}$ be a 2-face-colored rooted map on $n$ edges. Let $\nu, \epsilon, \phi$ be the permutations associated with $\mathscr{G}$ by the algorithm described above. Then $\mathscr{G}$ corresponds uniquely to the hypermap $(\nu, \epsilon)$. Conversely, each hypermap $(\nu, \epsilon)$ corresponds to a unique 2-face-colored map, up to a permutation of the color set. Moreover, $\phi=\nu \epsilon$.

The cycles of $\epsilon$ are called the hyperedges, and those of $\phi$ the hyperfaces, of the hypermap $(\nu, \epsilon)$.

For example, Figure 1 gives a 2 -colored rooted edge-labeled graph $\mathscr{G}$. The root edge of $\mathscr{G}$ is labeled 1 . The directions of the edges labeled $2,3, \ldots, 9$ are induced by the direction of the root edge. Clearly, $\nu=(2)(53)(7619)(48)$, $\epsilon=(12389)(4567)$, and $\phi=(125)(34)(6)(78)(9)$. The corresponding hypermap is therefore $((2)(53)(7619)(48),(12389)(4567))$.

Lemma 2.4. For each rooted map on $n$ edges there correspond $t^{\prime}(n)=(n-1)$ ! edge-labeled rooted maps.

Not every $(\nu, \epsilon) \in \mathfrak{S}_{n}^{2}$ corresponds to a hypermap, since $\nu$ and $\epsilon$ may not generate $\mathfrak{S}_{n}$. However, a combinatorial description can be given to every element of $\mathfrak{S}_{n}$. To this end, for $\rho, \sigma \in \mathfrak{S}_{N}$, let $\|\rho, \sigma\|$ denote the number of orbits of the group generated by $\rho$ and $\sigma$. For any $\mathscr{I} \subseteq \mathscr{N}_{n}$ and any $\mathscr{A}_{1}, \mathscr{A}_{2} \subseteq \mathscr{N}$, let $\mathscr{P}_{\mathscr{F}}^{\mathscr{H}_{1}}, \mathscr{A}_{2}$ be the set of all ordered pairs of permutations $(\rho, \sigma)$ on the elements of $\mathscr{I}$ such that $\rho$ has no cycles of length not in $\mathscr{A}_{1}$ and $\sigma$ has no cycles of length not in $\mathscr{A}_{2}$. Let $\mathscr{T}_{\mathscr{J}}^{\mathscr{A}_{1}, \mathscr{A}_{2}}=\left\{(\rho, \sigma) \in \mathscr{P}_{\mathscr{I}}^{\mathscr{H}_{1}, \mathscr{A}_{2}}:\|\rho, \sigma\|=1\right\}$. 
Corollary 2.5. $\mathscr{P}_{\mathscr{N}_{n}}^{\mathscr{A}_{1}, \mathscr{A}_{2}}$ is in 1-1 correspondence with $\mathscr{Q}_{n}^{\mathscr{A}_{1}, \mathscr{A}_{2}}$ where the latter is defined as

$$
\bigcup_{m=1}^{n}\left\{\left\{\left(\rho_{1}, \sigma_{1}\right), \ldots,\left(\rho_{m}, \sigma_{m}\right)\right\}: \sigma_{j} \in \mathscr{T}_{\mathscr{J}_{j}}^{\mathscr{A}_{1}, \mathscr{A}_{2}}, j=1, \ldots, m\right. \text {; }
$$

$$
\left.\mathscr{I}_{1} \dot{\cup} \cdots \dot{\cup} \mathscr{I}_{m}=\mathscr{N}_{n}\right\}
$$

Proof. For $(\rho, \sigma) \in \mathscr{P}_{\mathscr{N}_{n}}^{\mathscr{A}_{1}, \mathscr{A}_{2}}$, let $\mathscr{G}$ be the group generated by $\rho$ and $\sigma$. Suppose that $\mathscr{G}$ has orbits $\mathscr{I}_{1}, \ldots, \mathscr{I}_{m}$. Let $\rho_{j}$ be the permutation formed by the product of the cycles of $\rho$ which act on $\mathscr{I}_{j}$, for $j=1, \ldots, m$. Let $\sigma_{j}$ be the permutation formed by the product of the cycles of $\sigma$ which act on $\mathscr{J}_{j}$, for $j=1, \ldots, m$. Then $\left\{\left(\rho_{1}, \sigma_{1}\right), \ldots,\left(\rho_{m}, \sigma_{m}\right)\right\} \in \mathscr{Q}_{n}^{\mathscr{A}_{1}, \mathscr{A}_{2}}$. Let $\psi$ be the mapping

$$
\psi: \mathscr{P}_{\mathscr{N}_{n}}^{\mathscr{A}_{1}, \mathscr{A}_{2}} \rightarrow \mathscr{Q}_{n}^{\mathscr{A}_{1}, \mathscr{A}_{2}}:(\rho, \sigma) \mapsto\left\{\left(\rho_{1}, \sigma_{1}\right), \ldots,\left(\rho_{m}, \sigma_{m}\right)\right\} .
$$

Since $\left(\rho_{1}, \sigma_{1}\right), \ldots,\left(\rho_{m}, \sigma_{m}\right)$ all act on different sets, the products $\rho=\rho_{1} \ldots$ $\rho_{m}$ and $\sigma=\sigma_{1} \cdots \sigma_{m}$ are uniquely determined, and $(\rho, \sigma) \in \mathscr{P}_{\mathscr{N}_{n}}^{\mathscr{A}_{1}, \mathscr{A}_{2}}$. It is a straightforward matter to check that $\psi$ is bijective.

We conclude with a result which expresses important combinatorial information as a character sum.

Lemma 2.6. Let $\epsilon, \nu, \phi \vdash N$. Then

$$
\begin{gathered}
\left|\mathscr{C}_{\nu} \mathscr{C}_{\epsilon} \cap \mathscr{C}_{\phi}\right|=\frac{1}{N !} h^{\epsilon} h^{\nu} h^{\phi} \sum_{\theta \vdash N} \frac{1}{f^{\theta}} \chi_{\epsilon}^{\theta} \chi_{\nu}^{\theta} \chi_{\phi}^{\theta}, \\
\sum_{\alpha \vdash N} h^{\alpha} \chi_{\alpha}^{\theta} x^{l(\alpha)}=f^{\theta} H_{\theta}(x) .
\end{gathered}
$$

Proof. Direct from [5, Proposition 2.5, Lemma 2.6, Lemma 3.3].

\section{Generating functions}

Let $\mathscr{A}_{1}, \mathscr{A}_{2} \subseteq \mathscr{N}$, and let $x, y_{1}, y_{2}, z$ be independent commuting indeterminates. Let $\mathscr{P}_{\mathscr{A}_{1}, \mathscr{A}_{2}}\left(x, y_{1}, y_{2} \mid z\right)$ be the generating function for permutation systems $(\nu, \epsilon)$, of order 2 , in which $\nu \epsilon$ has no cycles of length not in $\mathscr{A}_{1}, \epsilon$ has no cycles of length not in $\mathscr{A}_{2}$, and $x, y_{1}, y_{2}$, and $z$ mark, respectively, the numbers of cycles in $\nu, \nu \epsilon, \epsilon \in \mathfrak{S}_{n}$, and $n$.

Similarly, let $C_{\mathscr{A}_{1}, \mathscr{A}_{2}}\left(u, x, y_{1}, y_{2}, z\right)$ be the generating function for rooted 2 -face-colored maps with no root-colored faces of degree not in $\mathscr{A}_{1}$, no nonrootcolored faces of degree not in $\mathscr{A}_{2}$, and in which $x, y_{1}, y_{2}, z$ mark, respectively, the number of vertices, faces of the root color, faces of the nonroot color, and edges; $u$ marks the genus.

In view of Lemma 2.4 and the Euler-Poincaré formula, it is convenient to introduce the mapping $\Theta_{u}$ defined by

$$
\Theta_{u}\left(x^{i} y_{1}^{j_{1}} y_{2}^{j_{2}} z^{k} / k !\right)=x^{i} y_{1}^{j_{1}} y_{2}^{j_{2}} z^{k} u^{2+k-i-j_{1}-j_{2}} / t^{\prime}(k)
$$


extended linearly to $\mathbf{Q}\left[\left[x, y_{1}, y_{2}, z\right]\right]$. We shall apply this only when $i, j_{1}, j_{2}$, $k$ count, respectively, the vertices, faces of the root color, faces of the nonroot color, and edges of a map so $2+k-i-j_{1}-j_{2}=2 g$ where $g$ is the genus of the map. The action of $\Theta_{u}$ on $f \in \mathbf{Q}\left[\left[x, y_{1}, y_{2}, z\right]\right]$ can be represented differentially by

$$
\Theta_{u} f\left(x, y_{1}, y_{2}, z\right)=u^{2} z \frac{\partial}{\partial z} f\left(x u^{-1}, y_{1} u^{-1}, y_{2} u^{-1}, z u\right)
$$

The operator $\Theta_{u}$ corresponds in the 2-colored rooted map (hypermap) case to the operator $\Omega_{u}$, defined in $\S 2$, for rooted maps.

\section{Lemma 3.1.}

$$
\begin{gathered}
C_{\mathscr{A}_{1}, \mathscr{A}_{2}}\left(u^{2}, x, y_{1}, y_{2}, z\right)=\Theta_{u} \log P_{\mathscr{A}_{1}, \mathscr{A}_{2}}\left(x, y_{1}, y_{2} \mid z\right), \\
P_{\mathscr{A}_{1}, \mathscr{A}_{2}}\left(x, y_{1}, y_{2} \mid z\right)=\sum_{n \geq 0} \frac{z^{n}}{(n !)^{2}} \sum_{\substack{\phi \in \Pi_{\mathscr{A}_{1}} \\
\epsilon \in \Pi_{\mathscr{d}_{2}}}} h^{\phi} y_{1}^{l(\phi)} h^{\epsilon} y_{2}^{l(\epsilon)} \sum_{\theta \vdash n} \chi_{\phi}^{\theta} \chi_{\epsilon}^{\theta} H_{\theta}(x) .
\end{gathered}
$$

Proof. (1) It follows from Corollary 2.5 that $\log \mathscr{P}_{\mathscr{A}_{1}, \mathscr{A}_{2}}$ is the generating function for edge-labeled 2-face-colored maps with the face restrictions specified by $\mathscr{A}_{1}, \mathscr{A}_{2}$. Thus, from Proposition 2.3 and Lemma 2.4, the generating function $C_{\mathscr{A}_{1}, \mathscr{A}_{2}}\left(u, x, y_{1}, y_{2}, z\right)$ for rooted 2 -face-colored maps is given by (1).

(2) By definition,

$$
P_{\mathscr{A}_{1}, \mathscr{A}_{2}}\left(x, y_{1}, y_{2} \mid z\right)=\sum_{n \geq 0} \frac{z^{n}}{n !} \sum_{\nu \vdash n} \sum_{\phi \in \Pi_{\mathscr{O}_{1}}} \sum_{\epsilon \in \Pi_{\mathscr{L}_{2}}} x^{l(\nu)} y_{1}^{l(\phi)} y_{2}^{l(\epsilon)}\left|\mathscr{C}_{\nu} \mathscr{C}_{\epsilon} \cap \mathscr{C}_{\phi}\right| .
$$

The result follows from Lemma 2.6.

Proposition 3.2. $M_{\mathscr{A}}\left(u, x, y_{1}, z^{2} y_{2}\right)=C_{\{2\}, \mathscr{A}}\left(u, x, y_{1}, y_{2}, z\right)$.

Proof. Given a rooted map, replace each edge by a digon. This operation is reversible and does not affect the genus.

To illustrate the methods of both this paper and [5], we include an algebraic proof of this result.

Proof (an algebraic proof of Proposition 3.2). Let

$$
J_{\mathscr{A}}\left(x, y_{2}\right)=\sum_{\epsilon \in \Pi_{\mathscr{A}}} h^{\epsilon} y_{2}^{l(\epsilon)} \sum_{\theta \vdash 2 n} \chi_{\left[2^{n}\right]}^{\theta} \chi_{\epsilon}^{\theta} H_{\theta}(x)
$$

Then, from Lemma 3.1,

$$
\begin{aligned}
C_{\{2\}, \mathscr{A}}\left(1, x, y_{1}, y_{2} \mid z\right) & =z \frac{\partial}{\partial z} \log P_{\{2\}, \mathscr{A}}\left(x, y_{1}, y_{2} \mid z\right) \\
& =z \frac{\partial}{\partial z} \log \sum_{n \geq 0} \frac{z^{2 n}}{(2 n) !} \frac{y_{1}^{n}}{2^{n} n !} J_{\mathscr{A}}\left(x, y_{2}\right) \\
& =\frac{\sum_{n \geq 0}\left(z^{2 n} /(2 n) !\right)\left(y_{1}^{n} / 2^{n-1}(n-1) !\right) J_{\mathscr{A}}\left(x, y_{2}\right)}{\sum_{n \geq 0}\left(z^{2 n} /(2 n) !\right)\left(y_{1}^{n} / 2^{n} n !\right) J_{\mathscr{A}}\left(x, y_{2}\right)} .
\end{aligned}
$$


Similarly, from [5, Corollary 3.5(2)],

$$
\begin{aligned}
M_{\mathscr{A}}(1, x, y, z) & =2 z \frac{\partial}{\partial z} \log \sum_{n \geq 0} \frac{z^{n}}{2^{n} n !(2 n) !} J_{\mathscr{A}}(x, y) \\
& =\frac{\sum_{n \geq 0}\left(2 z^{n} / 2^{n}(2 n) !(n-1) !\right) J_{\mathscr{A}}(x, y)}{\sum_{n \geq 0}\left(z^{n} / 2^{n}(2 n) ! n !\right) J_{\mathscr{A}}(x, y)} .
\end{aligned}
$$

Thus

$$
M_{\mathscr{A}}\left(1, x, y_{1}, z^{2} y_{2}\right)=\frac{\sum_{n \geq 0}\left(z^{2 n} y_{2}^{n} / 2^{n-1}(2 n) !(n-1) !\right) J_{\mathscr{A}}\left(x, y_{1}\right)}{\sum_{n \geq 0}\left(z^{2 n} y_{2}^{n} / 2^{n}(2 n) ! n !\right) J_{\mathscr{A}}\left(x, y_{1}\right)} .
$$

The result follows by duality, once the appropriate substitutions have been made to recover the genus.

The next corollary gives the generating function $P_{\mathscr{A}_{1}, \mathscr{A}_{2}}$ for some combinatorially useful choices of $\mathscr{A}_{1}$ and $\mathscr{A}_{2}$.

Lemma 3.3.

$$
P_{\mathscr{A}_{1}, \mathscr{N}}\left(x, y_{1}, y_{2} \mid z\right)=\sum_{n \geq 0} \frac{z^{n}}{(n !)^{2}} \sum_{\phi \in \Pi_{\mathscr{A}_{1}}} h^{\phi} y_{1}^{l(\phi)} \sum_{\theta \vdash n} f^{\theta} \chi_{\phi}^{\theta} H_{\theta}(x) H_{\theta}\left(y_{2}\right),
$$

$$
P_{\mathscr{N}, \mathscr{N}}\left(x, y_{1}, y_{2} \mid z\right)=\sum_{n \geq 0} \frac{z^{n}}{(n !)^{2}} \sum_{\theta \vdash n}\left(f^{\theta}\right)^{2} H_{\theta}(x) H_{\theta}\left(y_{1}\right) H_{\theta}\left(y_{2}\right)
$$

$$
P_{\{p\}, \mathscr{N}}\left(x, y_{1}, y_{2} \mid z\right)=\sum_{n \geq 0} \frac{z^{p n}}{(p n) !} \frac{y_{1}^{n}}{p^{n} n !} \sum_{\theta \vdash p n} f^{\theta} \chi_{\left[p^{n}\right]}^{\theta} H_{\theta}(x) H_{\theta}\left(y_{2}\right),
$$

$$
\begin{aligned}
& P_{\{p\},\{q\}}\left(x, y_{1}, y_{2} \mid z\right) \\
& \quad=\sum_{n \geq 0} z^{m n} \frac{y_{1}^{m_{1} n}}{p^{m_{1} n}\left(m_{1} n\right) !} \frac{y_{2}^{m_{2} n}}{q^{m_{2} n}\left(m_{2} n\right) !} \sum_{\theta \vdash m n} \chi_{\left[q^{m_{2} n}\right]}^{\theta} \chi_{\left[p^{m_{1} n}\right]}^{\theta} H_{\theta}(x),
\end{aligned}
$$

where $m=\operatorname{lcm}(p, q), m_{1}=m / p, m_{2}=m / q$, and $p$ and $q$ are positive integers.

Proof. Direct from Lemmas 3.1 and 2.6(2).

Under certain circumstances there are symmetries between the arguments $x$, $y_{1}, y_{2}$ of $P_{\mathscr{A}_{1}, \mathscr{A}_{2}}\left(x, y_{1}, y_{2} \mid z\right)$ and therefore the same arguments of $C_{\mathscr{A}_{1}, \mathscr{A}_{2}}$. These are evident combinatorially as well as algebraically from Lemma 3.3.

\section{Proposition 3.4.}

(1) $C_{\mathscr{N}, \mathscr{N}}\left(u, x, y_{1}, y_{2}, z\right)$ is a symmetric function in $x, y_{1}, y_{2}$;

(2) $C_{\mathscr{A}_{1}, \mathscr{N}}\left(u, x, y_{1}, y_{2}, z\right)$ is a symmetric function in $x, y_{2}$;

(3) $C_{\mathscr{A}_{1}, \mathscr{A}_{2}}\left(u, x, y_{1}, y_{2}, z\right)=C_{\mathscr{A}_{2}, \mathscr{A}_{1}}\left(u, x, y_{2}, y_{1}, z\right)$. 
In principle, it can be checked that $\left[u^{2 g}\right] \Theta_{u} \log P_{\mathscr{N}, \mathscr{N}}(1,1,1 \mid z)$ indeed gives the specializations of the known results for the sphere [8] and the torus [1], with $g=0$ and $g=1$, respectively.

\section{FACTORIZATIONS}

In this section we make use of factorizations of evaluations of $\chi^{\theta}$ at semiregular elements to obtain factorizations of the generating function for permutation systems. For this purpose, let $H_{j}^{\theta}(x)=\prod_{1 \leq i \leq l(\theta)}(x-j i+j)^{\left(j \theta_{i}\right)}$. The main result for this purpose [5, Lemma 4.8] is the following.

Lemma 4.1. Let $\Pi_{k, n}$ denote the set of all $k$-tuples $\left(\theta^{(1)}, \ldots, \theta^{(k)}\right)$ of partitions whose weights sum to $n$, and let $\mathscr{B}_{k, n}$ be the set of all $\theta \vdash k n$ such that $\chi_{\left[k^{n}\right]}^{\theta} \neq 0$. Then there exists a bijection

$$
\Delta_{k}: \mathscr{B}_{k, n} \rightarrow \Pi_{k, n}
$$

such that

$$
\chi_{\left[(k b)^{n}\right]}^{\theta}= \pm n ! \prod_{j=1}^{k} \frac{1}{n_{j} !} \chi_{\left[b^{n_{j}}\right]}^{\theta^{(j)}}
$$

for $\theta \in \mathscr{B}_{k, n}$, the sign depending only on $\theta$, where $\Delta_{k}(\theta)=\left(\theta^{(1)}, \ldots, \theta^{(k)}\right)$ and $n_{j}=\left|\theta^{(j)}\right| b^{-1}$.

Moreover,

$$
H_{1}^{\theta}(x)=H_{k}^{\theta^{(1)}}(x) H_{k}^{\theta^{(2)}}(x-1) \cdots H_{k}^{\theta^{(k)}}(x-k+1) .
$$

Many factorizations for permutation systems may be obtained. The one given relates to semiregular permutations and is used in $\S 5$ for combinatorial purposes.

Theorem 4.2. Let

$$
\widehat{A}\left(x, y_{1}, y_{2} \mid z\right)=\sum_{n \geq 0} \frac{\left(p^{p r-r-1} z^{p r} y_{1}^{r} y_{2}\right)^{n}}{n ! r^{n}} A_{\left[r^{n}\right]}^{(p)}\left(\frac{x}{p}, \frac{x+1}{p}, \ldots, \frac{x+p-1}{p}\right) .
$$

Then

$$
P_{\{p\},\{p r\}}\left(x, y_{1}, y_{2} \mid z\right)=\widehat{A}\left(x, y_{1}, y_{2} \mid z\right) \cdots \widehat{A}\left(x-p+1, y_{1}, y_{2} \mid z\right) .
$$

Proof. For brevity, let $a$ denote the generating function $P_{\{p\},\{p r\}}\left(x, y_{1}, y_{2} \mid z\right)$. Then from Lemma 3.3(4)

$$
a=\sum_{n \geq 0} \frac{z^{p r n} y_{1}^{r n} y_{2}^{n}}{p^{r n}(r n) !(r p)^{n} n !} \sum_{\theta \vdash p r n} \chi_{\left[(p r)^{n}\right]}^{\theta} \chi_{\left[p^{r n}\right]}^{\theta} H_{\theta}(x) .
$$

By applying $\Delta_{p}$ and by rearranging the sum we have, from Lemma 4.1, 


$$
\begin{aligned}
a= & \sum_{n \geq 0} \frac{z^{p r n} y_{1}^{r n} y_{2}^{n}}{p^{r n}(r n) !(r p)^{n} n !} \sum_{\substack{s_{1}, \ldots, s_{p} \geq 0 \\
s_{1}+\cdots+s_{p}=n}}\left(\begin{array}{c}
n \\
s_{1}, \ldots, s_{p}
\end{array}\right)\left(\begin{array}{c}
r n \\
r s_{1}, \ldots, r s_{p}
\end{array}\right) \\
& \cdot \sum \prod_{j=1}^{p} f^{\theta^{(j)}} \chi_{\left[r^{r_{j}}\right]}^{\theta^{(j)}} H_{p}^{\theta^{(j)}}(x-j+1)
\end{aligned}
$$

where the third summation is over all $\left(\theta^{(1)}, \ldots, \theta^{(p)}\right)$ such that $\theta^{(j)} \vdash r s_{j}$ for $j=1, \ldots, p$. Thus

$$
a=\widehat{A}\left(x, y_{1}, y_{2} \mid z\right) \cdots \widehat{A}\left(x-p+1, y_{1}, y_{2} \mid z\right)
$$

where

$$
\widehat{A}\left(x, y_{1}, y_{2} \mid z\right)=\sum_{|\theta| \equiv 0 \bmod r} \frac{z^{p|\theta|} y_{1}^{|\theta|} y_{2}^{|\theta| / r}}{p^{|\theta|}(|\theta| / r) !(r p)^{|\theta| / r}|\theta| !} f^{\theta} \chi_{\left[r^{|\theta| / r}\right]}^{\theta} H_{p}^{\theta}(x) .
$$

The result now follows by rearrangement of the series.

The two specific instances of Theorem 4.2 which are given in Corollary 4.3 have been selected since they lead to bijections between natural classes of rooted maps.

Corollary 4.3. Let

$$
\begin{aligned}
& U(x)=P_{\{r\}, \mathscr{N}}\left(\frac{1}{2} x, \frac{1}{2} y_{2}, \frac{1}{2}(x+1) \mid 2 z^{2} y_{1}\right), \\
& T(x)=P_{\mathscr{N}, \mathscr{N}}\left(\frac{1}{3} x, \frac{1}{3}(x+1), \frac{1}{3}(x+2) \mid 3 z^{3} y_{1} y_{2}\right) .
\end{aligned}
$$

Then

$$
\begin{gathered}
P_{\{2\},\{2 r\}}\left(x, y_{1}, y_{2} \mid z\right)=U(x) U(x-1), \\
P_{\{3\},\{3\}}\left(x, y_{1}, y_{2} \mid z\right)=T(x) T(x-1) T(x-2) .
\end{gathered}
$$

Proof. (1) When $p=2$ then

$$
\widehat{A}\left(x, y_{1}, y_{2} \mid z\right)=P_{\{r\}, \mathscr{N}}\left(\frac{1}{2} x, \frac{1}{2} y_{2}, \frac{1}{2}(x+1) \mid 2 z^{2} y_{1}\right)
$$

and (1) now follows from Theorem 4.2.

(2) When $p=3$ and $r=1$ then

$$
\widehat{A}\left(x, y_{1}, y_{2} \mid z\right)=P_{\mathscr{N}, \mathscr{N}}\left(\frac{1}{3} x, \frac{1}{3}(x+1), \frac{1}{3}(x+2) \mid 3 z^{3} y_{1} y_{2}\right)
$$

and (2) follows from Theorem 4.2.

\section{FACE-COLORED MAPS OF ARBITRARY GENUS}

We are now in a position to give combinatorial applications of the factorizations for permutation systems. 
Corollary 5.1. Let $C_{\mathscr{A}_{1}, \mathscr{A}_{2}}^{(g)}\left(x, y_{1}, y_{2}, z\right)=\left[u^{2 g}\right] C_{\mathscr{A}_{1}, \mathscr{A}_{2}}\left(u^{2}, x, y_{1}, y_{2}, z\right)$. Then

$$
\begin{gathered}
M_{\{2 r\}}\left(u^{2}, x, y, z\right)= \\
\frac{1}{2} C_{\{r\}, \mathscr{N}}\left(4 u^{2}, x+u, y, x, z\right) \\
+\frac{1}{2} C_{\{r\}, \mathscr{N}}\left(4 u^{2}, x-u, y, x, z\right), \\
M_{\{2 r\}}^{(g)}(x, y, z)=\left.\sum_{i=0}^{g} \frac{4^{i}}{(2 g-2 i) !} \frac{\partial^{2 g-2 i}}{\partial x^{2 g-2 i}} C_{\{r\}, \mathscr{N}}^{(i)}\left(x, y_{1}, y_{2}, z\right)\right|_{\substack{y_{1} \mapsto y \\
y_{2} \mapsto x}} .
\end{gathered}
$$

Proof. (1) From Lemma 3.1

$$
C_{\{2\},\{2 r\}}\left(u^{2}, x, y_{1}, y_{2}, z\right)=u^{2} z \frac{\partial}{\partial z} \log P_{\{2\},\{2 r\}}\left(x u^{-1}, y_{1} u^{-1}, y_{2} u^{-1}, z u\right) \text {. }
$$

Let $C_{\{2\},\{2 r\}}$ denote the left-hand side of this equation. Then, from Corollary 4.3(1),

$$
\begin{aligned}
C_{\{2\},\{2 r\}}= & u^{2} z \frac{\partial}{\partial z} \log P_{\{r\}, \mathscr{N}}\left(\frac{1}{2} x u^{-1}, \frac{1}{2} y_{2} u^{-1}, \frac{1}{2}\left(x u^{-1}+1\right) \mid 2 z^{2} u y_{1}\right) \\
& +u^{2} z \frac{\partial}{\partial z} \log P_{\{r\}, \mathscr{N}}\left(\frac{1}{2}\left(x u^{-1}-1\right), \frac{1}{2} y_{2} u^{-1}, \frac{1}{2} x u^{-1} \mid 2 z^{2} u y_{1}\right) .
\end{aligned}
$$

Let $\log P_{\{r\}, \mathscr{N}}\left(x, y_{1}, y_{2} \mid z\right)=\sum_{i, j_{1}, j_{2}, k \geq 0} c_{i, j_{1}, j_{2}, k} x^{i} y_{1}^{j_{1}} y_{2}^{j_{2}} z^{k} / k !$. Then

$$
\begin{aligned}
C_{\{2\},\{2 r\}}= & \sum_{i, j_{1}, j_{2}, k \geq 0} c_{i, j_{1}, j_{2}, k} \frac{z^{2 k}}{(k-1) !} y_{1}^{k} 2^{k-j_{1}-j_{2}+1} u^{k-j_{1}-j_{2}+2} y_{2}^{j_{1}} \\
& \cdot\left(x^{i}(x+u)^{j_{2}}+(x-u)^{i} x^{j_{2}}\right) \\
= & \frac{1}{2} C_{\{r\}, \mathscr{N}}\left(4 u^{2}, x, y_{2}, x+u, z^{2} y_{1}\right) \\
& +\frac{1}{2} C_{\{r\}, \mathscr{N}}\left(4 u^{2}, x-u, y_{2}, x, z^{2} y_{1}\right)
\end{aligned}
$$

since $c_{i, j_{1}, j_{2}, k} /(k-1)$ ! counts rooted maps of genus $g$ where $2 g=k-i-$ $j_{1}-j_{2}+2$. The result now follows from Propositions 3.2 and 3.4.

(2) This is immediate from (1).

The result [5, Corollary 5.2] indicating a bijection for rooted quadrangulations of arbitrary genus is recovered by setting $r=2$ in Corollary 5.1 and using Proposition 3.2.

Setting $g=0$ gives a generating function equation indicating a bijection between rooted $2 r$-face-regular maps on the sphere and rooted 2-colored maps on the sphere such that all root-colored faces have degree $r$. This bijection is a modification of the construction for the medial (e.g., $[5, \S 5])$. Let $\mu$ be a 2-colored map with all the faces of one color (white) having degree $r$. Now put a new vertex in each of the faces of the other color (shaded). Connect the new vertices to each of the vertices in the boundary of their faces. Remove the edges of $\mu$ to obtain a new map $\mu^{*}$. This map is $2 r$-face-regular. An example is given in Figure 2 for the case $r=3$. 


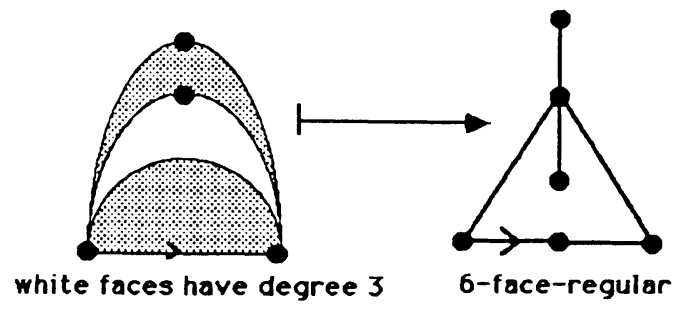

FIGURE 2. Example of the modified medial construction

\section{Corollary 5.2.}

$$
\begin{aligned}
3 C_{\{3\},\{3\}} & \left(u^{2}, x, y_{1}, y_{2}, z\right)=C_{\mathscr{N}, \mathscr{N}}\left(9 u^{2}, x, x+u, x+2 u, z^{3} y_{1} y_{2}\right) \\
& +C_{\mathscr{N}, \mathscr{N}}\left(9 u^{2}, x-u, x, x+u, z^{3} y_{1} y_{2}\right) \\
& +C_{\mathscr{N}, \mathscr{N}}\left(9 u^{2}, x-2 u, x-u, x, z^{3} y_{1} y_{2}\right) .
\end{aligned}
$$

Proof. Let $C_{\{3\},\{3\}}$ denote $C_{\{3\},\{3\}}\left(u^{2}, x, y_{1}, y_{2}, z\right)$. Then from Lemma 3.1 and Corollary $4.3(2)$,

$$
\begin{aligned}
C_{\{3\},\{3\}}= & u^{2} z \frac{\partial}{\partial z} \log P_{\{3\},\{3\}}\left(x u^{-1}, y_{1} u^{-1}, y_{2} u^{-1} \mid z u\right) \\
= & u^{2} z \frac{\partial}{\partial z} \log P_{\mathscr{N}, \mathscr{N}}\left(\frac{1}{3} x u^{-1}, \frac{1}{3}\left(x u^{-1}+1\right), \frac{1}{3}\left(x u^{-1}+2\right) \mid 3 z^{3} u y_{1} y_{2}\right) \\
& +u^{2} z \frac{\partial}{\partial z} \log P_{\mathscr{N}, \mathscr{N}}\left(\frac{1}{3}\left(x u^{-1}-1\right), \frac{1}{3} x u^{-1}, \frac{1}{3}\left(x u^{-1}+1\right) \mid 3 z^{3} u y_{1} y_{2}\right) \\
& +u^{2} z \frac{\partial}{\partial z} \log P_{\mathscr{N}, \mathscr{N}}\left(\frac{1}{3}\left(x u^{-1}-2\right), \frac{1}{3}\left(x u^{-1}-1\right), \frac{1}{3} x u^{-1} \mid 3 z^{3} u y_{1} y_{2}\right) .
\end{aligned}
$$

Let $\log P_{\mathscr{N}, \mathscr{N}}\left(x, y_{1}, y_{2} \mid z\right)=\sum_{i, j_{1}, j_{2}, k \geq 0} d_{i, j_{1}, j_{2}, k} x^{i} y_{i}^{j_{1}} y_{2}^{j_{2}} z^{k} / k !$. The proof is completed in a way analogous to that of Corollary 5.1. The details are omitted.

When $g=0$ we obtain a generating function equation corresponding to the known bijection [9] between rooted 2-colored triangulations on the sphere and rooted 2-colored maps on the sphere. An example of this bijection is given in Figure 3 and is defined as follows. Let $\mu$ be a rooted 2-colored map. The image of $\mu$ under this bijection is the union of the dual $\hat{\mu}$ of $\mu$ and the medial $\mu^{*}$. To see that this is invertible, it is sufficient to note that the root vertex of $\hat{\mu} \cup \mu^{*}$ is the root vertex of $\mu$. Since $\hat{\mu} \cup \mu^{*}$ is bipartite, the block $\mathscr{Y}$ of the associated vertex partition of $\hat{\mu} \cup \mu^{*}$ not containing the root vertex is the vertex set of $\hat{\mu}$. Thus $\hat{\mu}$ is recoverable as the subgraph of $\hat{\mu} \cup \mu^{*}$ vertex-induced by $\mathscr{Y}$. The remaining edges of $\hat{\mu} \cup \mu^{*}$ are the edges of $\mu^{*}$, and $\mu$ and its root edge are recoverable.

Tutte [9] used the term bicubic for the duals of 2-colored rooted maps and used the bijection for the sphere in an early approach to the enumeration of planar maps. 


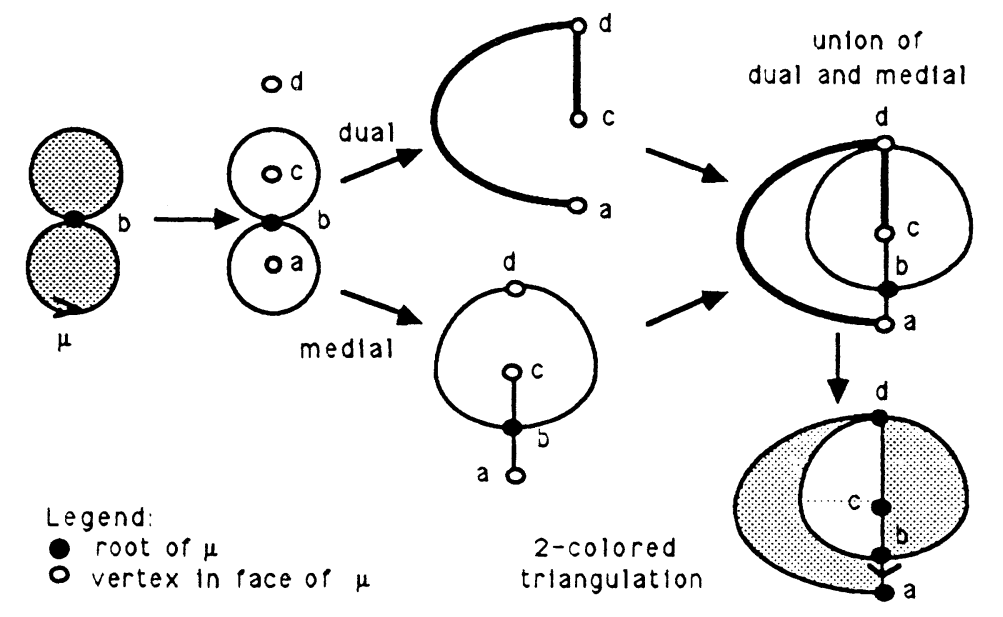

FiguRE 3. A 2-colored map and the corresponding 2-colored triangulation

\section{CONCLUding COMMENTS}

Decompositions of rooted maps in surfaces of arbitrary genus are uncommon. It would therefore be of interest to have purely combinatorial explanations of Corollaries 5.1 and 5.2, particularly in the light of [5, Corollary 5.2].

There may be a natural combinatorial interpretation of permutation systems of order greater than two.

\section{REFERENCES}

1. D. Arquès, Hypercartes pointées sur le tore: décompositions et dénombrements, J. Combinatorial Theory Ser. B 84 (1987), 275-286.

2. R. Cori, Un code pour les graphes plainaires et ses applications, Thèse de Doctorat, Paris, 1973.

3. J. L. Gross and T. W. Tucker, Topological graph theory, Wiley Interscience, New York, 1987.

4. D. M. Jackson, Some combinatorial problems associated with products of conjugacy classes of the symmetric group, J. Combinatorial Theory Ser. A 49 (1988), 363-369.

5. D. M. Jackson and T. I. Visentin, A character theoretic approach to embeddings of rooted maps in an orientable surface of given genus, Trans. Amer. Math. Soc. 322 (1990), 343-363.

6. I. G. Macdonald, Symmetric functions and Hall polynomials, Clarendon Press, Oxford, 1979.

7. J.-P. Serre, Linear representations of finite groups, Springer-Verlag, New York, 1977.

8. W. T. Tutte, $A$ census of slicings, Canad. J. Math. 14 (1962), 708-722.

9. __ A census of planar maps, Canad. J. Math. 15 (1963), 249-271.

10. T. R. S. Walsh, Hypermaps versus bipartite maps, J. Combinatorial Theory Ser. B 18 (1975), 155-163.

Department of Combinatorics and Optimization, University of Waterloo, Waterloo, Ontario, Canada N2L 3G1 